\title{
Efficient Objective Metric Tool for Medical Electrical Device Development: Eye Phantom for Glaucoma Diagnosis Device
}

\author{
Anthony Deluthault, ${ }^{1}$ Luc Mezenge, ${ }^{2}$ Philippe Cauvet, ${ }^{2}$ Vincent Kerzérho, ${ }^{1}$ \\ Fabien Soulier, ${ }^{1}$ and Serge Bernard ${ }^{1}$ \\ ${ }^{1}$ LIRMM/CNRS Laboratory, University of Montpellier, 161 rue Ada, 34095 Montpellier Cedex 5, France \\ ${ }^{2}$ Ophtimalia SAS, 2 Esplanade Anton Philips, Campus Effiscience, 14460 Colombelles, France \\ Correspondence should be addressed to Serge Bernard; serge.bernard@lirmm.fr
}

Received 9 September 2016; Revised 18 March 2017; Accepted 29 March 2017; Published 1 June 2017

Academic Editor: Fanli Meng

Copyright (C) 2017 Anthony Deluthault et al. This is an open access article distributed under the Creative Commons Attribution License, which permits unrestricted use, distribution, and reproduction in any medium, provided the original work is properly cited.

\begin{abstract}
Developing electronic medical devices is challenging. Simulations or in vivo experiments are not sufficient to obtain pertinent comparisons between potential design options. This paper presents a new artificial tool allowing objective comparisons between electronic device topologies. The main idea is to build a tool which is sensitive to targeted biological parameters only. These tools are generally called phantoms. The phantom presented in this paper is dedicated to IntraOcular Pressure (IOP) Measurement devices used in glaucoma diagnosis and treatment. It is called Biomechanical Eye Emulator (BEE). The BEE emulates the main biomechanical parameters influencing the IOP measurements. Because it is not sensitive to the living context, the BEE is the most efficient tool to investigate the best sensor design. BEE specifications are defined to be as close as possible to chosen models (humans or animals). Its efficiency is shown with a case study on rabbits. The results clearly demonstrate the BEE phantoms efficacy in providing objective assessment metrics during the sensor design process.
\end{abstract}

\section{Introduction}

Medical devices are intended for treating patients or facilitating the diagnosis of pathologies with minimal risks for patients. Similar to other fields of application such as avionics and automotive industry, medical devices integrate an increasing number of electronic components which must meet strict dependability requirements. Thus, accurately qualifying electronic medical devices is an important step towards maximal dependability.

The term e-Health designates a relatively recent healthcare practice based on electronics and communication. In the e-Health context, patients are equipped with sensors linked with an electronic device. The sensors may be non- or minimally invasive or even invasive.

The developing of electronic medical devices requires more than only simulations. Indeed, living context models are not accurate enough to integrate all the parameters involved in the relationship between the electronic device and the living environment. Unfortunately, validating or qualifying the electronic medical devices in vivo creates several issues:

(i) The Variability of Both Physiological and Anatomic Parameters of a Given Population. Electronic medical devices are designed to measure one or several physiological or anatomical parameters. Unfortunately, human beings or animals are made of a wide variety of such parameters. Moreover, the interaction between these parameters usually generates uncontrollable effects. Consider blood pressure: two subjects can exhibit different values at the same time because of multiple factors such as age, weight, and temperature. Comparing two technological solutions by measuring the same parameter on two different living subjects thus becomes irrelevant.

(ii) The Lack of Stability and Repeatability of the Measurements. Measurement repeatability is a critical factor when developing any measuring instrument. 
Indeed, the same measurement is repeated on one population sample in order to qualify the device under development. Subsequently, several statistical parameters, such as average and standard deviation, are computed and analyzed. However, in vivo conditions (e.g., study on animals), variations in the environment may significantly bias the results. For both economical and ethical reasons, ex vivo experiments are often conducted before in vivo tests. Under ex vivo conditions, although setups can be easily controlled, tissue characteristics are rapidly altered (coagulation, desiccation, necrosis, etc.), leading to measurement shifts and therefore consequently to biases.

As repeatability and accuracy cannot be ensured during in vivo or ex vivo experiments, making pertinent comparisons between potential design solutions is very challenging. In order to fill the gap between conventional validation tools (simulators and classic test benches) and the ex vivo or in vivo experiments, an interesting solution consists in artificially replicating living organs [1]. These solutions are called phantoms. The objective of phantoms is not to emulate the complete behavior of organs but only to mimic the main parameters which influence the device's performance. This "intermediate" solution meets the requirements for achieving the lowest cost, the shortest setup time, the best repeatability, and the highest reliability. In this method, living tissue variations do not interfere with the validations, and accurate and fast comparisons of various solutions can be conducted for device design. This solution is particularly suitable for the development of systems whose performance is governed by a limited number of living parameters.

In this paper, we propose an eye phantom, called the Biomechanical Eye Emulator (BEE), to be used as objective metric tool for the design of electrical devices for glaucoma diagnosis.

Section 2 presents the BEE's characteristics. The validation process of this new tool is then described in Section 3 and further illustrated with a rabbit eye emulation example.

\section{Biomechanical Eye Emulator (BEE)}

2.1. Introduction. Glaucoma is an optic neuropathy damaging the optic nerve. The damage is permanent and leads to blindness if left untreated. This pathology is usually associated with increased aqueous humor pressure in the eye (Figure 1) inducing an abnormally high IntraOcular Pressure (IOP). In order to insure the monitoring of IOP variations during a period of at least 24 hours, Ophtimalia, a company, is developing an e-Health device. The basic operating condition consists in monitoring the deformation of the cornea with a sensor embedded in a lens $[2,3]$. The system is composed of three elements $[4,5]$ : the contact lens, the eyewear, and the electronic recorder. The contact lens embeds a passive sensor as a resonant circuit, which is stimulated by the eyewear electronics. The lens deformation induces a variation of the sensor resonant frequency that is detected through magnetic coupling by the antenna on the eyewear. In this context, we want to develop an objective tool to the design phase of this

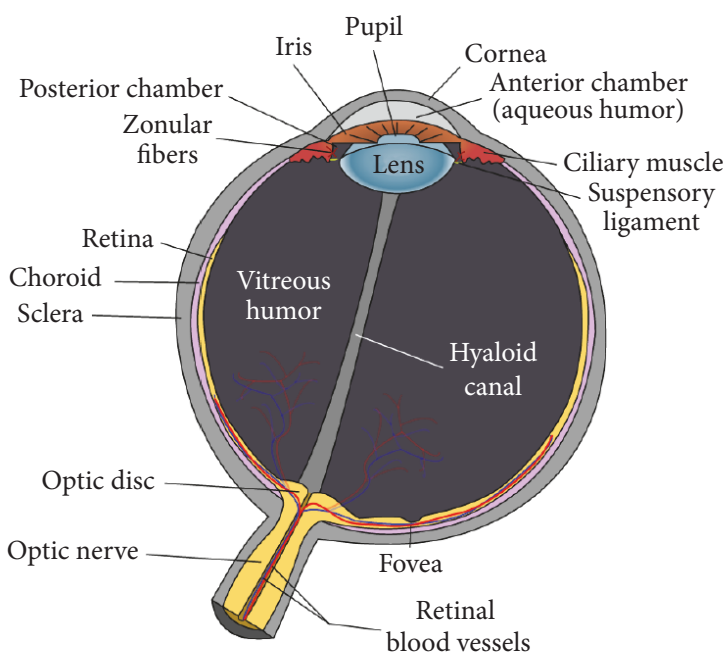

Figure 1: Schematic of human eye.

sensor in order to be able to compare various design options. Figure 2 shows the development flow of such devices, up to industrialization, with or without the BEE. As described in Figure 2, the BEE replicates the usual biomechanical behavior patterns of the cornea, introducing key parameters that can be critical for in vivo experimentation, such as thickness or size of the cornea (which may differ from humans to animals).

2.2. Eye Phantom State of the Art. Phantoms are devices designed to emulate one or several characteristics of the living world and are generally developed for biomedical research. Their design is driven by the motivation to overcome the drawbacks of in vivo and ex vivo experiments, such as the potential uncontrollable variability of experimental conditions or the harmful effects. Phantoms have been widely studied by medical imaging researchers [6]. Indeed, the need for phantoms has risen with the discovery of the harmful sideeffects of radiation doses. Since phantom design is strongly constrained by the device's purpose, many different imaging phantoms have been developed. Their shapes can be square (water-tank phantoms) or more complex (anthropomorphic phantoms). Historically, the materials used to simulate the tissue are water, wax, or wood [6]. Over time, several new materials, such as epoxy, have been used because of their greater reproducibility of industrial manufacturing processes [6]. Some phantoms even contain human bones in order to improve their body simulation characteristics.

In the current context of ophthalmic applications, several eye phantoms exist. In 2005, Yoriyaz et al. [7] presented a mathematical human eye model for dosimetry purposes. Indeed, radiotherapy may be used for the treatment of eye tumors. This phantom was designed to determine the dose according to various therapies. The mathematical model integrates the different elements of the eye: cornea, sclera, optic nerve, vitreous body, and so forth. It also includes the tumor model. The model takes into account the dimensions and masses of the elements. While this model is suitable for dosimetry applications, to our knowledge, there is no model 


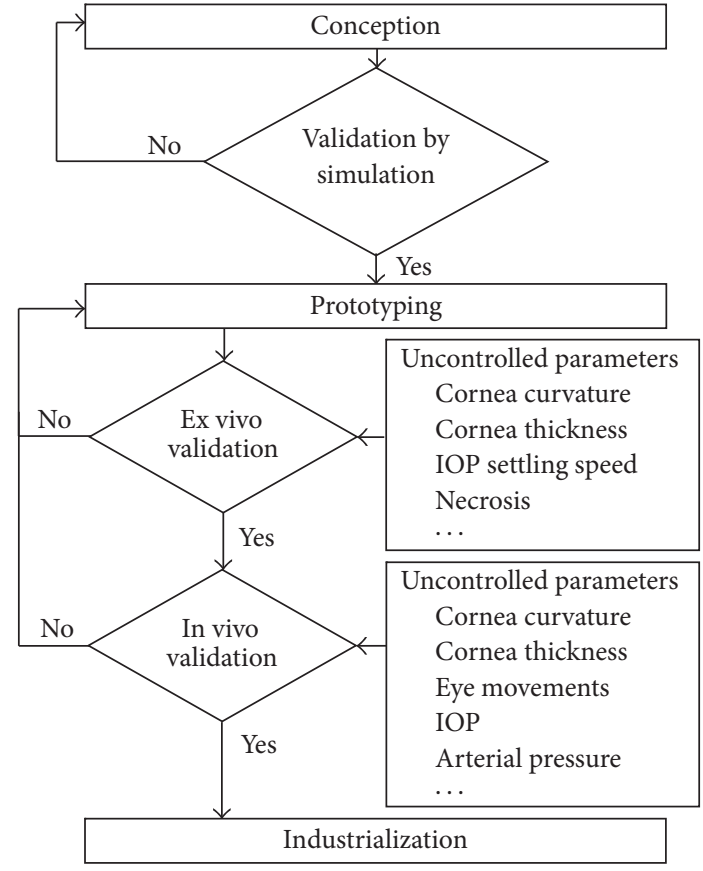

(a)

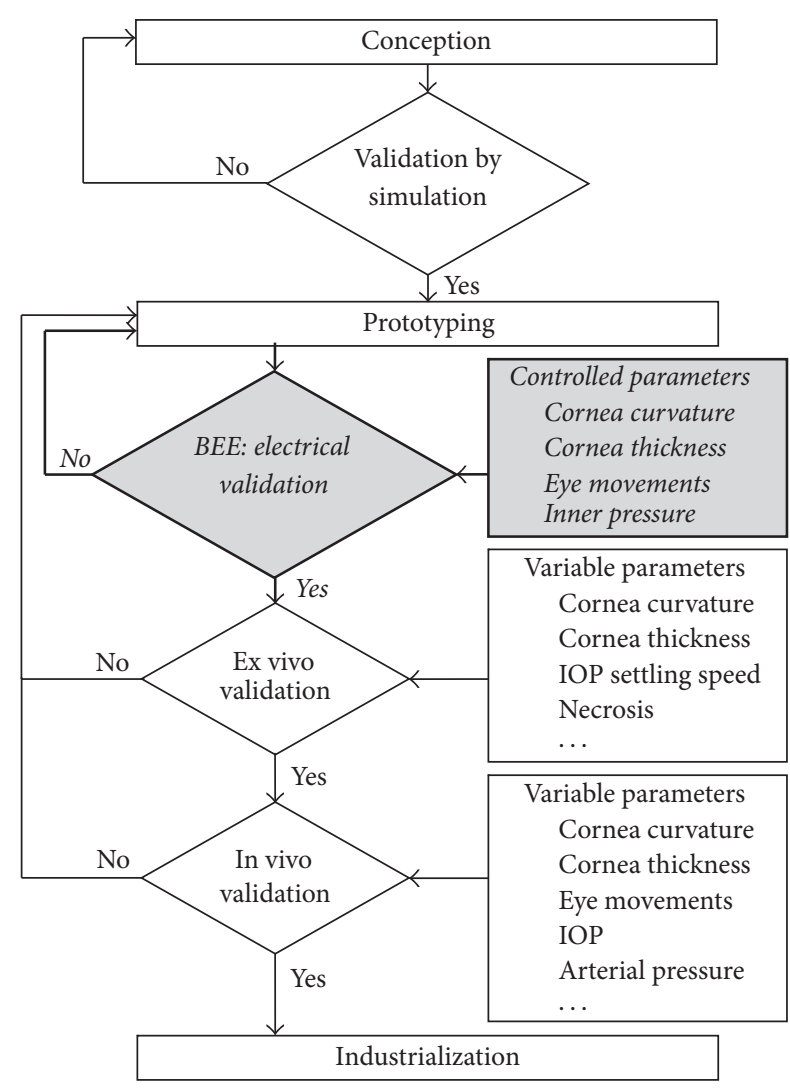

(b)

FIGURE 2: Development flow of lens-based IOP variation monitoring systems without (a) or with (b) the BEE.

for measuring IOP variations and their impact on cornea strain.

Oxygen saturation is a relevant biomarker for monitoring the ocular health. Measurements can be made with a video camera. In 2009 [8], Lemaillet and Ramella-Roman designed an eye phantom to calibrate such a measurement instrument. This eye phantom is made of a rectangular mechanical housing to simulate the eyeball, with a $6 \mathrm{~mm}$ hole for the pupil. A microtube is used as a retinal blood vessel. The eye fundus is mimicked by a multilayer structure. According to the context of oximetry measurement using a camera, these elements were chosen for their own wavelength-dependent absorption and scattering properties. This eye phantom mimics oximetry, a dynamic characteristic of the eye, but this physiological characteristic is not the phenomenon that has to be emulated in our context.

Another optical technique used for diagnosis is the optical coherence tomography (OCT). OCT is interesting because it is notably used to diagnose glaucoma. In this case, OCT is used to measure the nerve's fiber layer thickness. According to Baxi et al. [9], the variability and nonrepeatability of measurements using various OCT instruments demonstrates the need for a controlled test object (i.e., an eye phantom). The authors presented a retina-mimicking phantom, which incorporates all retinal layers visible with current OCT systems. The layers are made of polydimethylsiloxane.
The specificity of this phantom is its detailed and accurate replication of retina tissue. Unfortunately, this phantom is specific to the OCT application and cannot be used in our context.

Laukhin et al. [10] presented the first eye phantom developed for testing lens-based IOP measurement systems. In this phantom, the lens-based sensor is placed on a glass adapter. The air pressure inside the glass adapter is controlled by a low pressure transducer enabling changes from 0 to $22 \mathrm{mmHg}$. Beyond its pioneering contribution in ocular field, this phantom is remarkable in that it helps validate the concept of measuring pressure variations with lens sensor for a pressure range similar to the one in the human eyes. It can also emulate some disruptive phenomena, such as the ocular pulse. Unfortunately, this eye phantom cannot mimic the cornea strain caused by IOP. Without this information it is impossible to estimate the real impact of IOP on the cornea distortion, which is essential for Ophtimalia's sensor.

2.3. BEE Architecture. This section details the architecture and characteristics of the Biomechanical Eye Emulator (BEE), as presented in Figure 3. It is mainly composed of two parts, the artificial ocular globe and the control system. The anatomical emulation of an eye by the BEE is performed by an artificial ocular globe. This globe is made of two parts, shown in Figure 4. First, it consists of half sphere made 


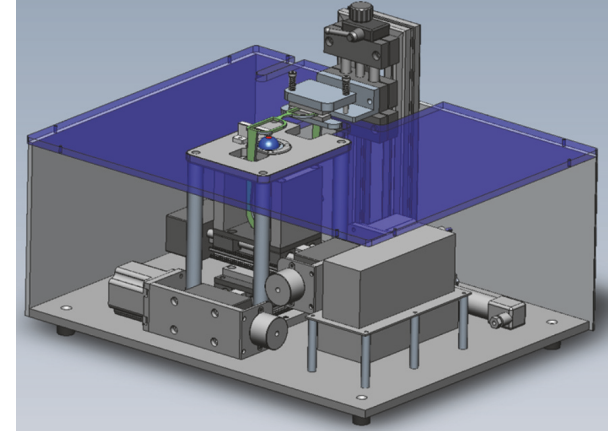

Figure 3: The Biomechanical Eye Emulator system.

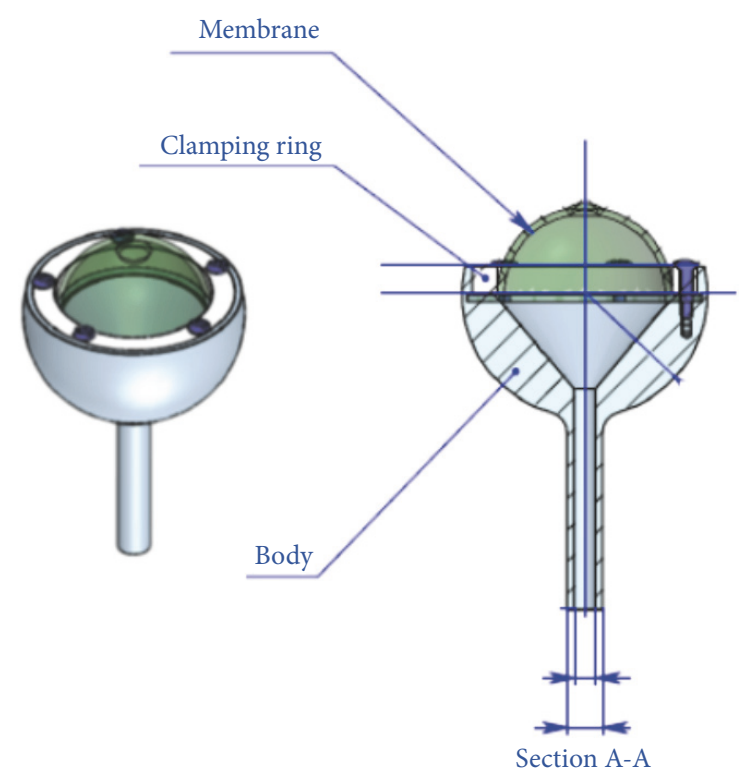

Figure 4: Artificial ocular globe.

of steel designed to emulate the posterior globe of the eye. The entire ocular globe can sustain deformation with IOP increase (the cornea and the sclera are both deformationsensitive). Because the cornea is more sensitive to IOPinduced deformation, we have chosen a rigid material to exclusively replicate the biomechanical behavior patterns of the cornea [11,12]. The second part of the globe emulates the anterior chamber; that is, mainly the cornea and part of the sclera are both made of one consolidated silicone membrane. This membrane can be changed, to reproduce the human or animal variability of characteristics such as cornea thickness, rigidity or size. A silicone material was chosen to allow for strain under pressure increases. The characteristics of the membrane are described in detail in the following subsection.

The physiomechanical control of the artificial ocular globe relies on two parts: pressure control via a syringe pump and motion control via two stepper motors.

The pressure within the artificial ocular globe varies with the amount of water injected in the rear part. The water is injected using the syringe pump (KD Scientific, Microliter OEM, Model \#78-2900).
The artificial ocular globe is placed on a motionless support which allows its rotation. The two rotation degrees of freedom are insured by two stepper motors (NEMA $23^{\circledR}$ Danaher).

Additionally, a serial pressure sensor is used to monitor the pressure inside the BEE.

A user interface has been developed using Labview ${ }^{\circledR}$. It is used to program the operation and outcome of the stepper motors, the syringe pump, and the pressure sensor (see Figure 5).

2.4. BEE Specifications. The BEE specifications must include several key in vivo parameters, such as cornea size or thickness, with the aim of replicating the global biomechanical pattern.

Firstly, the specifications ranges are chosen to be as close as possible to human variability. These features are shown in Table 1.

The human values from Table 1 were collected from the literature.

Because of the nonuniform radius and thickness of the cornea, we decided to focus on the central part of the cornea for human radius and thickness characteristics. The value of human cornea radius value is an average of values found in the literature [13]. The range of central human corneal thickness is considered as normal [13-17].

The cornea is composed of different layers and the stroma represents about $90 \%$ of the corneal thickness. Consequently, we consider that the cornea is mainly composed of collagen for the values in Table 1 [11]. It represents the most prevalent corneal element in its mechanical behavior with its interwoven collagen lamellae $[18,19]$.

The human eye pressure range covers the IOP of healthy patients and IOP of subjects with different types of glaucoma $[13-15,17]$.

The maximum rotation velocity is found in saccade cases, where the velocity depends on the movement's amplitude. Studies show that the velocity can exceed $400^{\circ} / \mathrm{s}$ [20-22].

The anatomical characteristics to target are those of the cornea emulated by the silicone membrane. In order to emulate the IOP-induced strains of the cornea (see Figure 4), the membrane has been shaped like the front of the eye.

The choice of the membrane material is also important. Silicone was selected because it is massively used in the industry. Silicone exhibits linear cornea-like deformation due to IOP for a low pressure range (around 0 to $20 \mathrm{mmHg}$ ).

The ultimate objective is to emulate usual biomechanical behavior patterns, so pertinent comparisons between different pressure sensor designs can be made. Reproducing the high-complexity structure of nonuniform stiffness and deformation is difficult, particularly when human variability is to be factored in. For instance, corneal deformation hysteresis may not be assessed with this tool because the frequency of the IOP variations is too low for the BEE's pressure sensor capability in terms of measurement time.

According to [11], Young's modulus can be extracted from experimentation on the cornea. Its value is related to the IOP and to the age of the patient. For an average central corneal thickness of $500 \mu \mathrm{m}$, the modulus can be 3 to 12 times lower 


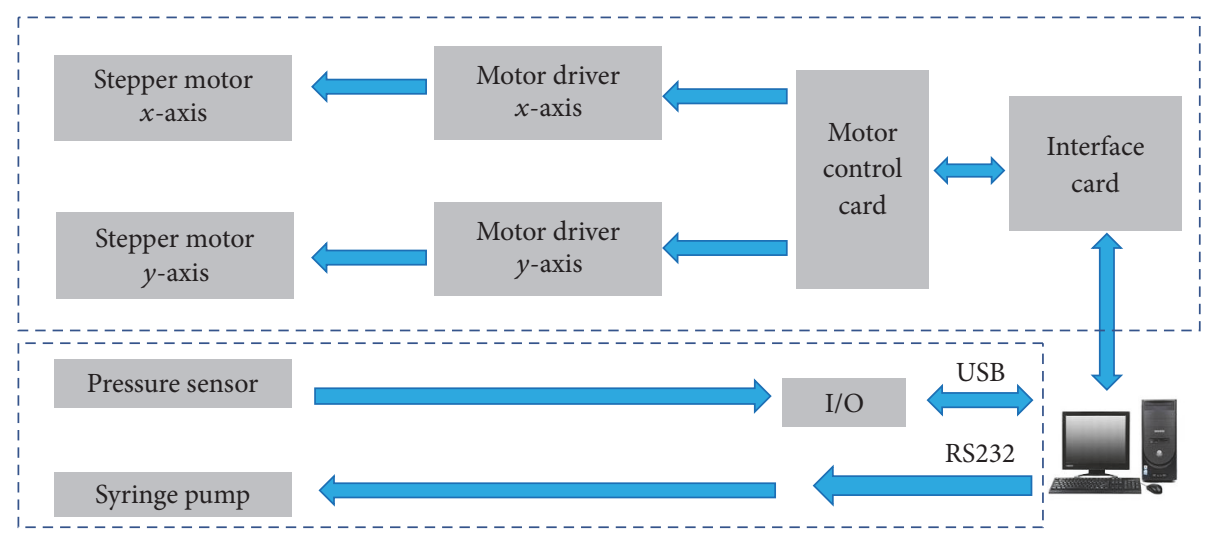

FIgURE 5: Architecture system.

TABLE 1: BEE and human features.

\begin{tabular}{lcc}
\hline & BEE features & $\begin{array}{c}\text { Human } \\
\text { characteristics }\end{array}$ \\
\hline Anatomical & & \\
$\quad$ Cornea radius & $\sim 8.2 \mathrm{~mm}$ & $7.6 \mathrm{~mm}$ \\
Central membrane thickness & $\sim 210 \mu \mathrm{m}$ & $500-600 \mu \mathrm{m}$ \\
Membrane/human material & Silicone & Collagen fibrils \\
Material Young's modulus & $\sim 2.5 \mathrm{MPa}$ & $<0.2-0.8 \mathrm{MPa}$ \\
Physiological & & \\
Pressure range & $10-50 \mathrm{mmHg}$ & $10-30 \mathrm{mmHg}$ \\
Pressure precision & $\sim 0,09 \mathrm{mmHg}$ & \\
Rotation range & $0,1-30^{\circ}$ & $0-45^{\circ}$ \\
Maximum rotation velocity & $187^{\circ} / \mathrm{s}$ & $>400^{\circ} / \mathrm{s}$ \\
\hline
\end{tabular}

than Young's modulus of the silicone used in the artificial ocular globe. To replicate the average stiffness of a real eye for an IOP ranging from 10 to $40 \mathrm{mmHg}$, a membrane thickness 3 to 12 times thinner than the central corneal thickness is necessary. For the BEE, because of manufacturing limitations, membrane thickness was set to 3 times thinner.

According to Table 1, several specifications do not match with the human variability. This is mainly due to limitations intrinsic to industrial manufacturing process. The aim of the BEE is not to create an exact copy of the human eye, but to provide a tool. This tool is an enhancement compared to regular electronic test benches. In addition it avoids drawbacks of in vivo experimentations. This is the reason why another silicone membrane, with different specifications, may be substituted to match another variability range (human or animal). Only key parameters are taken into account. Further parameters and functions can be added in future system versions.

\section{BEE Validation}

3.1. Architecture Validation. This part aims to validate the correct output control of the BEE.

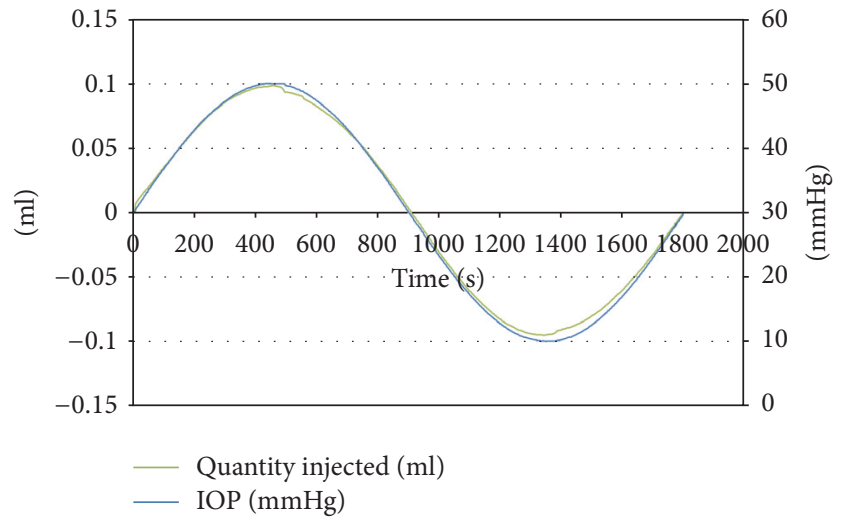

FIgURE 6: Pressure variation according to quantity of water injected by the syringe pump over time. The gray curve represents the artificial ocular globe pressure $(\mathrm{mmHg})$ and the black curve shows the quantity of water injected $(\mathrm{ml})$.

Two experiments were conducted to validate the correct control of the artificial ocular globe pressure of the BEE. The first experimentation aimed to check the correlation between the injected water and the pressure measured inside the artificial ocular globe. The pressure was measured by the inner serial sensor.

The variation of the water volume follows a sine shape. The water quantity is controlled by the syringe pump, starting with an initial quantity of water around $10 \mathrm{ml}$ (for a pressure of $30 \mathrm{mmHg}$ ) and varying from 9.9 to $10.1 \mathrm{ml}$.

Figure 6 shows the varying quantity of water injected and the pressure variation detected by the sensor. The pressure variation ranges from 10 to $50 \mathrm{mmHg}$.

The resulting pressure, plotted as a function of the amount of injected water, is shown in Figure 7 and reveals a linear response with a narrow spread.

The purpose of the second experimentation is to compare the pressure measured by the inner sensor and the one measured by a tonometer, an external instrument dedicated to IOP measurements. Because of the horizontal position of the BEE system, the most efficient and practical tonometer is the TonoPen ${ }^{\circledR}$. Indeed, we need portable device to be able to 


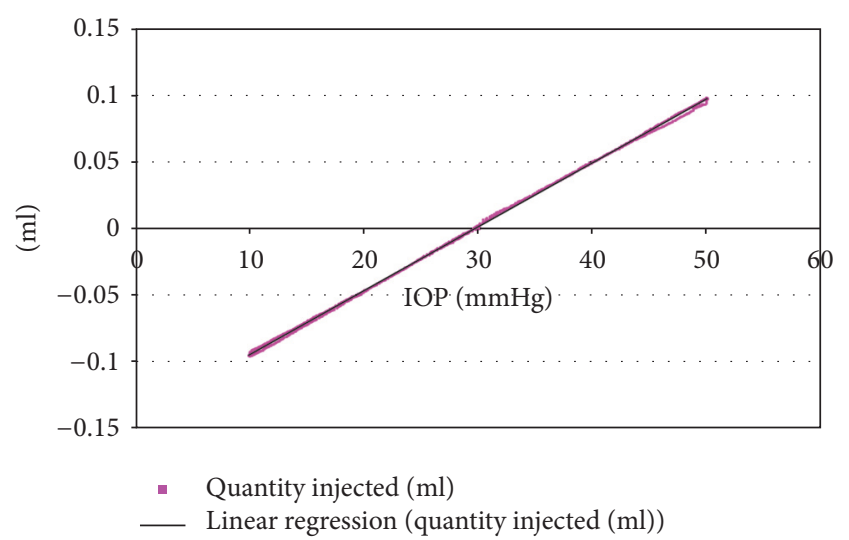

FIGURE 7: Quantity of water injected versus pressure measured. The gray points represent the quantity of water injected $(\mathrm{ml})$ and the black curve the linearized quantity of water injected $(\mathrm{ml})$.

TABLE 2: Comparison: measurements made by BEE's pressure sensor and by a TonoPen.

\begin{tabular}{lc}
\hline $\begin{array}{l}\text { Artificial ocular globe pressure } \\
(\mathrm{mmHg})\end{array}$ & $\begin{array}{c}\text { TonoPen's pressure } \\
(\mathrm{mmHg})\end{array}$ \\
\hline 20 & 21 \\
31 & 27 \\
40 & 34 \\
\hline
\end{tabular}

remove the instrument when we use our sensor and we need instrument useable on the vertical position. From existing tonometers only the TonoPen combines these capabilities and above all, it is a standard reference instrument widely used by ophthalmologists. Table 2 shows the measurements taken with a TonoPen and the measurements of the inner pressure sensor of the BEE system. The measurements were performed using a membrane thickness of $200 \mu \mathrm{m}$.

Though a slight difference with the TonoPen results was observed, the pressure inside the artificial ocular globe was adequately generated and monitored by the BEE's control and sensor. We believe the differences between the TonoPen and the BEE's inner sensor results are due to the TonoPen. Indeed the latter usually underestimates measurements over $20 \mathrm{mmHg}[23,24]$.

3.2. Case Study Validation. The objective is to compare topologies or sensor solutions on both their sensitivity to IOP variations and their robustness to use and parasitic parameters. In this section, we illustrate the use of the BEE with a real case of IOP measurement on a rabbit. Figure 8 shows the lens held by the BEE. Because this step aims to identify the best design for future in vivo experimentations on rabbits, the membrane dimensions are changed to fit the corneal features of a twelve month-old rabbit; see Table 3 . The corneal features have been taken from the literature $[25,26]$.

The first step consists in comparing two prototypes of lens-based IOP variation measurement systems called Lens $A$ and Lens $B$. These prototypes are based on different sensor architectures to convert eye-lens deformation to resonance

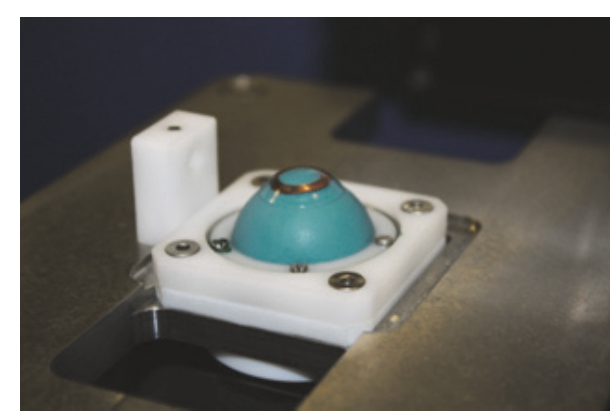

Figure 8: A contact lens on the artificial ocular globe.

TABLE 3: Comparison: rabbit corneal features and membrane sizes.

\begin{tabular}{lcc}
\hline & BEE features & $\begin{array}{c}\text { Rabbit } \\
\text { characteristics }\end{array}$ \\
\hline Cornea curvature & $7.3 \mathrm{~mm}$ & $7.26 \pm 0.26 \mathrm{~mm}$ \\
Cornea vertical diameter & $14.08 \mathrm{~mm}$ & $13.02 \pm 0.3 \mathrm{~mm}$ \\
Cornea horizontal diameter & $14.8 \mathrm{~mm}$ & $13.41 \pm 0.34 \mathrm{~mm}$ \\
\hline
\end{tabular}

frequency variations. A first experiment consists in varying the artificial ocular globe pressure of the BEE while making a continuous measurement of the pressure with the prototypes. Artificial tears are used between the lens and the artificial ocular globe to obtain as a realistic response. Tears are, in fact, emulated by a thin coat of glycerin. The pressure within the artificial ocular globe variations follows a sine shape ranging from $25 \mathrm{mmHg}$ to $35 \mathrm{mmHg}$. Figures 9 and 10 show the IOP variations and the resonance frequency measured by the system.

The first sensor's performance we want to estimate is the sensitivity. The sensitivity is the ratio between resonant frequency and IOP variations. With a better sensitivity, a sensor has a larger resonant frequency variation for a given IOP variation. For Lens $A$ prototype, the sensitivity is very low (lower than $5 \mathrm{kHz} / \mathrm{mmHg}$ ). It is difficult to see the IOP variations on the resonance frequency response. With Lens $B$ prototype, we obtain a good correlation between resonance frequency and the pressure within the artificial ocular globe. The sensitivity is four times higher $(20 \mathrm{kHz} / \mathrm{mmHg})$ than for Lens A. However, the resonance frequency measurements are affected by a drift effect. This effect is due to the evacuation of the liquid placed between the artificial ocular globe and the lens. This experiment highlights the high sensitivity of Lens B's frequency to contextual humidity and a design fault in Lens $A$. These issues would have not been readily identified without the BEE.

Even if the sensitivity of Lens $B$ is sufficient, the measurement drift is an issue for devices used over 24 hours. Based on this first result, another sensor architecture was designed. This new architecture was made less sensitive to contextual humidity. For this design, we reused part of the topology of Lens $B$ to maximize its sensitivity. This new sensor, called Lens $C$, has a resonance frequency around $80 \mathrm{MHz}$. The new experiment consists in varying the pressure within the artificial ocular globe variations following a sine shape 


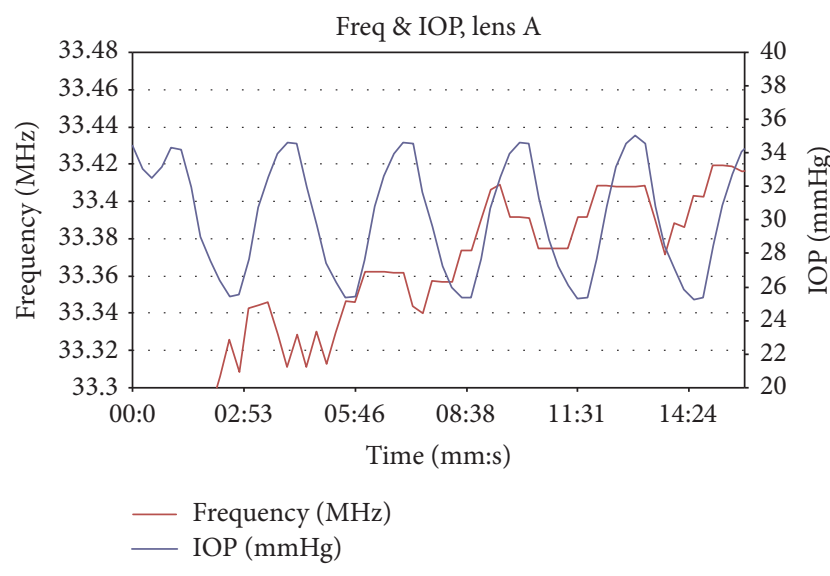

FIGURE 9: Pressure variations in the BEE and variations of the related resonance frequency given by the Ophtimalia's pressure sensor Lens $A$ over time. The blue curve represents the artificial ocular globe pressure $(\mathrm{mmHg})$ and the red curve shows the frequency $(\mathrm{Hz})$.

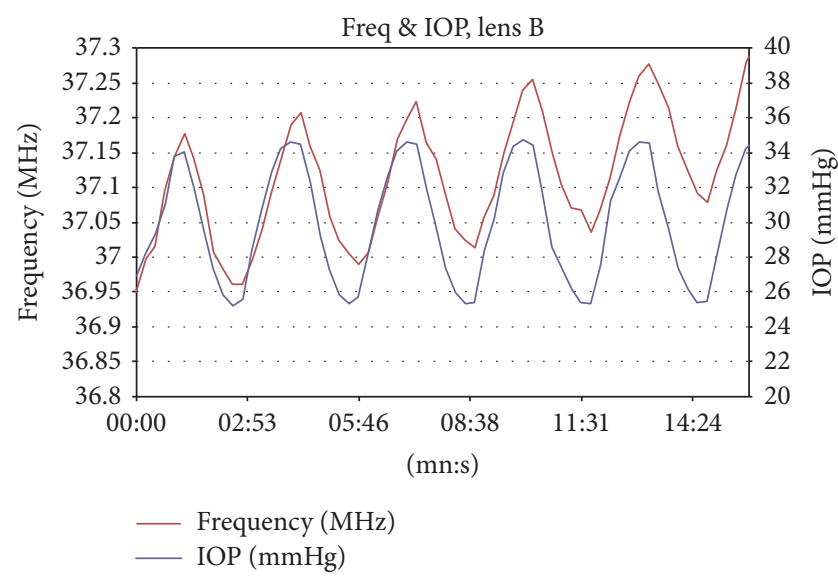

FIGURE 10: Pressure variations in the BEE and variations of the related resonance frequency given by Ophtimalia's pressure sensor Lens $B$ over time. The blue curve represents the artificial ocular globe pressure $(\mathrm{mmHg})$ and the red curve shows the frequency $(\mathrm{Hz})$.

between $15 \mathrm{mmHg}$ and $25 \mathrm{mmHg}$. The sensitivity, equal to $10 \mathrm{kHz} / \mathrm{mmHg}$, is lower than Lens $B$, but the results shown in Figure 11 demonstrate the accuracy of this new architecture and the lack of drift effect. The correlation between actual pressure and the resonance frequency shows $R^{2}$ higher than 0.94, as presented in Figure 12.

Several major biological or biomechanical parameters were taken into account in these experiments. The results allow us to select the best architecture in terms of sensitivity and robustness. The design process of measurement devices is often complex and involves many trade-offs. Finding the best balanced solution means designing a tool allowing replicable and objective comparisons between potential solutions. Once again, such observations are impossible under in vivo conditions and only systems like the BEE allow replicable measurements. This case study demonstrated that the BEE is an efficient tool to design e-Health sensors.

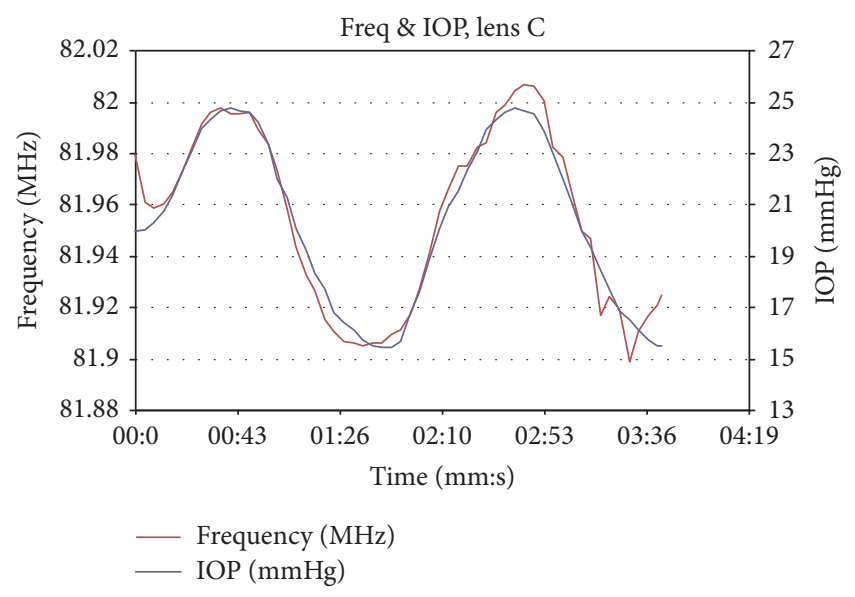

FIGURE 11: Pressure variations in the BEE and variations of the related resonance frequency given by Ophtimalia's pressure sensor Lens $C$ over time. The blue curve represents the artificial ocular globe pressure $(\mathrm{mmHg})$ and the red curve shows the frequency $(\mathrm{Hz})$.

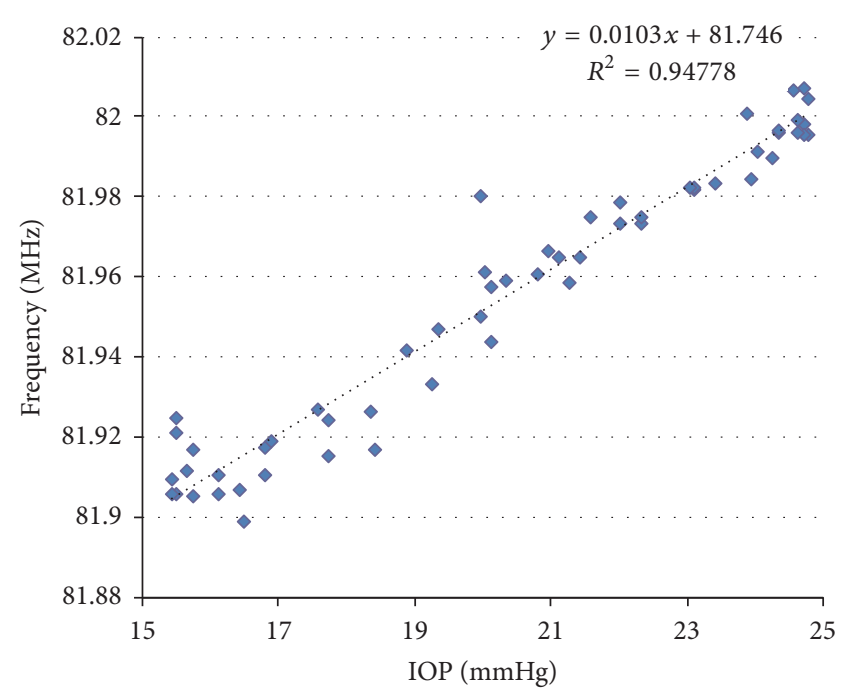

FIgURE 12: Correlation between the pressure in the BEE and of the related resonance frequency given by the Ophtimalia's pressure sensor Lens $C$.

\section{Discussion}

As demonstrated above, the BEE is an efficient tool to obtain objective metrics for comparing various design options in electrical device development. Nevertheless, phantoms are not perfect (i.e., complete) emulators of biomechanical behaviors. The approach to design an artificial test bench from a living target therefore has limitations. Living aspects include many physiological and anatomical parameters, which are weakly or strongly correlated with what we aim to monitor.

In our application, we focused on the parameters most correlated with the biomechanical parameters of our IOP sensor. But additional features should be implemented in the next version of the BEE: 
(i) From an anatomical standpoint, the representation of the cornea and the sclera were simplified and reduced to a single element. In future work, this part can be split to distinguish the cornea from the sclera. Two chambers and the iris can be added to simulate aqueous humor flow management within the ocular globe (see Figure 1) more adequately. A new membrane with nonuniform thickness and made of a material whose composition and stiffness closer to the cornea should be used.

(ii) The addition of an eyelid, as well as blinking, may affect ocular pressure balance as measured in IOP monitoring when using contact lenses.

(iii) From a physiological standpoint, the phenomenon due to blood pressure and associated with the heart rate produces slight IOP variations called Ocular Pulse [17].

In conclusion, all existing or additional parameters and functions must cover the whole spectrum of human variability. The closer we seek to be to a real eye, the more anatomical and physiological features or functions we must add. Nonetheless, the first aim of this system was not to make a copy of a real eye but to provide a scientific, replicable comparison basis during the design phase of e-Health devices. In order to use it like a test bench and enhance the development of solutions, we must agree to certain trade-offs in the selection of critical parameters for the BEEs first version.

\section{Conclusion}

As explained above, the aim of the BEE is not to provide an artificial copy of an eye, but a test bench to enable a controlled and pertinent comparison between e-Health device architectures. The objective is to emulate bioparameters detected by the device. In vivo experiments remain necessary to validate the efficacy of an IOP variation measurement system before its commercialization. The BEE has a metallic body and uses a membrane to emulate the cornea and a mechanical system to control the motions of the artificial eye. The results presented in this paper highlight the BEEs efficacy in allowing objective comparisons between various sensor options during the design process. This type of tool is an interesting solution to successfully complete the first steps of sensor design in medical applications.

\section{Conflicts of Interest}

The authors declare that they have no conflicts of interest.

\section{Acknowledgments}

The authors acknowledge the support of the Agence National de la Recherche (French National Research Agency) under Reference ANR-11-INSE-16, SACSO.

\section{References}

[1] L. N. S. Andreasen, J. J. Struijk, and M. K. Haugland, "An artificial nerve fiber for evaluation of nerve cuff electrodes," Engineering in Medicine and Biology Society, pp. 1997-1999, 1997.

[2] G. Lissorgues, L. Rousseau, P. Poulichet et al., "Continuous intra ocular pressure measurement sensor for glaucoma diagnostic," International Ophthalmology, pp. 1281-1285, 2010.

[3] P. Auvray, L. Rousseau, G. Lissorgues et al., "A passive pressure sensor for continuously measuring the intraocular pressure in glaucomatous patients," IRBM, vol. 33, no. 2, pp. 117-122, 2012.

[4] P. Auvray, P. Biemans, P. Cauvet, and F. Verjus, "Patent: Systme multidiagnostic sans contact utilisant des paramtres physiologiques oculaires," Ophtimalia EP 10290 034.7, 2011.

[5] G. L. P. Auvray and L. Rousseau, "Patent: Capteur passif flexible intgr? dans une lentille de contact souple pour surveillance iop," Ophtimalia EP 10290 429.9, 2012.

[6] L. DeWerd and M. Kissick, The Phantoms of Medical and Health Physics: Devices for Research and Development, seriesBiological and Medical Physics, Biomedical Engineering, Springer, London, UK, 2013, http://books.google.fr/books.

[7] H. Yoriyaz, A. Sanchez, and A. dos Santos, "A new human eye model for ophthalmic brachytherapy dosimetry," Radiation Protection Dosimetry, vol. 115, no. 1-4, pp. 316-319, 2005.

[8] P. Lemaillet and J. C. Ramella-Roman, "Dynamic eye phantom for retinal oximetry measurements," Journal of Biomedical Optics, vol. 14, no. 6, Article ID 064008, 2009.

[9] J. Baxi, W. Calhoun, Y. J. Sepah et al., "Retina-simulating phantom for optical coherence tomography," Journal of Biomedical Optics, vol. 19, no. 2, Article ID 021106, 2014.

[10] V. Laukhin, I. Sánchez, A. Moya et al., "Non-invasive intraocular pressure monitoring with a contact lens engineered with a nanostructured polymeric sensing film," Sensors and Actuators, A: Physical, vol. 170, no. 1-2, pp. 36-43, 2011.

[11] A. Elsheikh, Understanding Corneal Biomechanics Through Experimental Assessment and Numerical Simulation, Eye and Vision Research Developments Series, 2010.

[12] S. L.-Y. Woo, A. S. Kobayashi, W. A. Schlegel, and C. Lawrence, "Nonlinear material properties of intact cornea and sclera," Experimental Eye Research, vol. 14, no. 1, pp. 29-39, 1972.

[13] P. Gunvant, M. Baskaran, L. Vijaya et al., "Effect of corneal parameters on measurements using the pulsatile ocular blood flow tonograph and goldmann applanation tonometer," British Journal of Ophthalmology, vol. 88, no. 4, pp. 518-522, 2004.

[14] A. C. Sobottka Ventura, M. Böhnke, and D. S. Mojon, "Central corneal thickness measurements in patients with normal tension glaucoma, primary open angle glaucoma, pseudoexfoliation glaucoma, or ocular hypertension," British Journal of Ophthalmology, vol. 85, no. 7, pp. 792-795, 2001.

[15] A. P. Wells, D. F. Garway-Heath, A. Poostchi, T. Wong, K. C. Y. Chan, and N. Sachdev, "Corneal hysteresis but not corneal thickness correlates with optic nerve surface compliance in glaucoma patients," Investigative Ophthalmology and Visual Science, vol. 49, no. 8, pp. 3262-3268, 2008.

[16] M. Detry-Morel, "Utilité de la pachymétrie cornéenne dans l'hypertension oculaire et le glaucome chronique," The Bulletin of the Belgian Society of Ophthalmology, vol. 293, pp. 1-9, 2004.

[17] C. Kaufmann, L. M. Bachmann, Y. C. Robert, and M. A. Thiel, "Ocular pulse amplitude in healthy subjects as measured by dynamic contour tonometry," Archives of Ophthalmology, vol. 124, no. 8, pp. 1104-1108, 2006. 
[18] K. Anderson, A. El-Sheikh, and T. Newson, "Application of structural analysis to the mechanical behaviour of the cornea," Journal of the Royal Society Interface, vol. 1, no. 1, pp. 3-15, 2004.

[19] A. Elsheikh, D. Alhasso, and P. Rama, "Assessment of the epithelium's contribution to corneal biomechanics," Experimental Eye Research, vol. 86, no. 2, pp. 445-451, 2008.

[20] A. L. Yarbzls, Eye Movements and Vision, Plenum Press, 1967.

[21] H. Sasaki and N. Ishii, "Study on the velocity of saccadic eye movements," in Knowledge-Based Intelligent Information and Engineering Systems, R. Khosla, R. J. Howlett, and L. C. Jain, Eds., vol. 3681 of Lecture Notes in Computer Science, pp. 808812, Springer, Berlin, Germany, 2005.

[22] R. A. Abrams, D. E. Meyer, and S. Kornblum, "Speed and accuracy of saccadic eye movements: characteristics of impulse variability in the oculomotor system," Journal of Experimental Psychology: Human Perception and Performance, vol. 15, no. 3, pp. 529-543, 1989.

[23] M. Iester, A. Mermoud, F. Achache, and S. Roy, "New Tonopen XL: comparison with the Goldmann tonometer," Eye, vol. 15, no. 1, pp. 52-58, 2001.

[24] V. Hessemer, R. Rössler, and K. W. Jacobi, “Tono-Pen, a new tonometer," International Ophthalmology, vol. 13, no. 1-2, pp. 51$56,1989$.

[25] M. J. Doughty, "The cornea and corneal endothelium in the aged rabbit," Optometry and Vision Science, vol. 71, no. 12, pp. 809818, 1994.

[26] G. Bozkir, M. Bozkir, H. Dogan, K. Aycan, and B. Guler, Measurements of Axial Length and Radius of Corneal Curvature in The Rabbit Eye, Okayama university of medical school, 1997. 


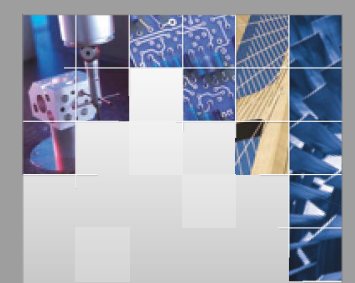

\section{Enfincering}
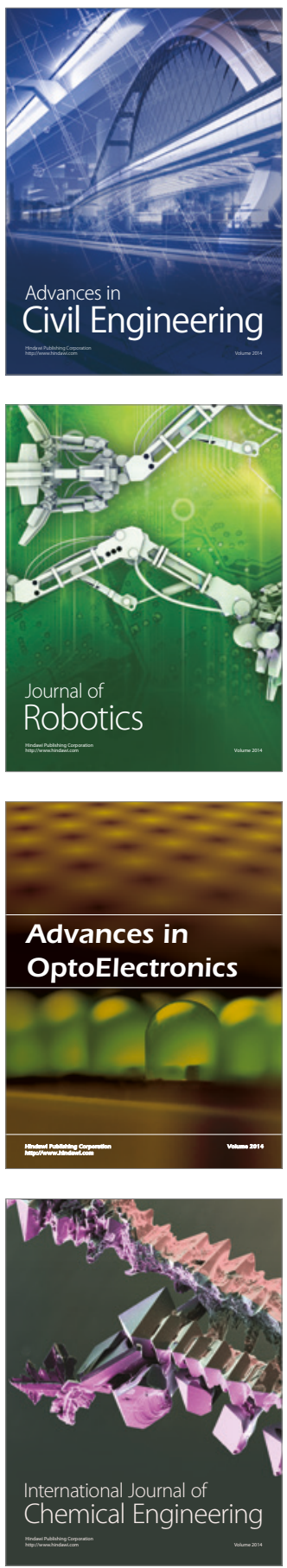

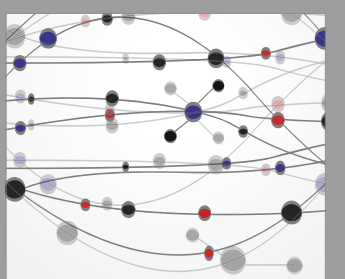

The Scientific World Journal

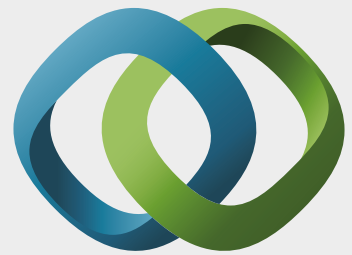

\section{Hindawi}

Submit your manuscripts at

https://www.hindawi.com
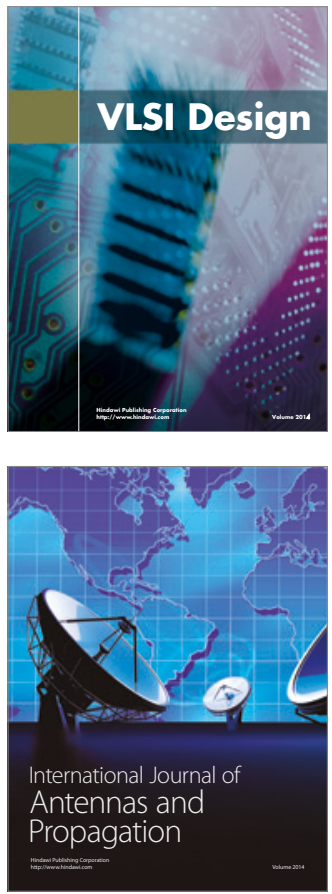

\section{Rotating}

Machinery
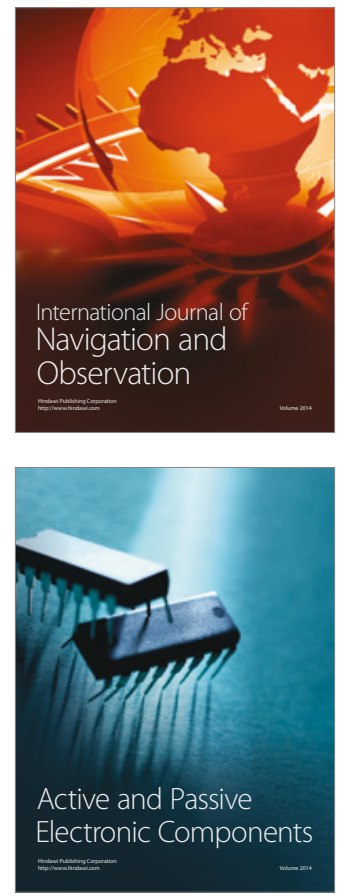
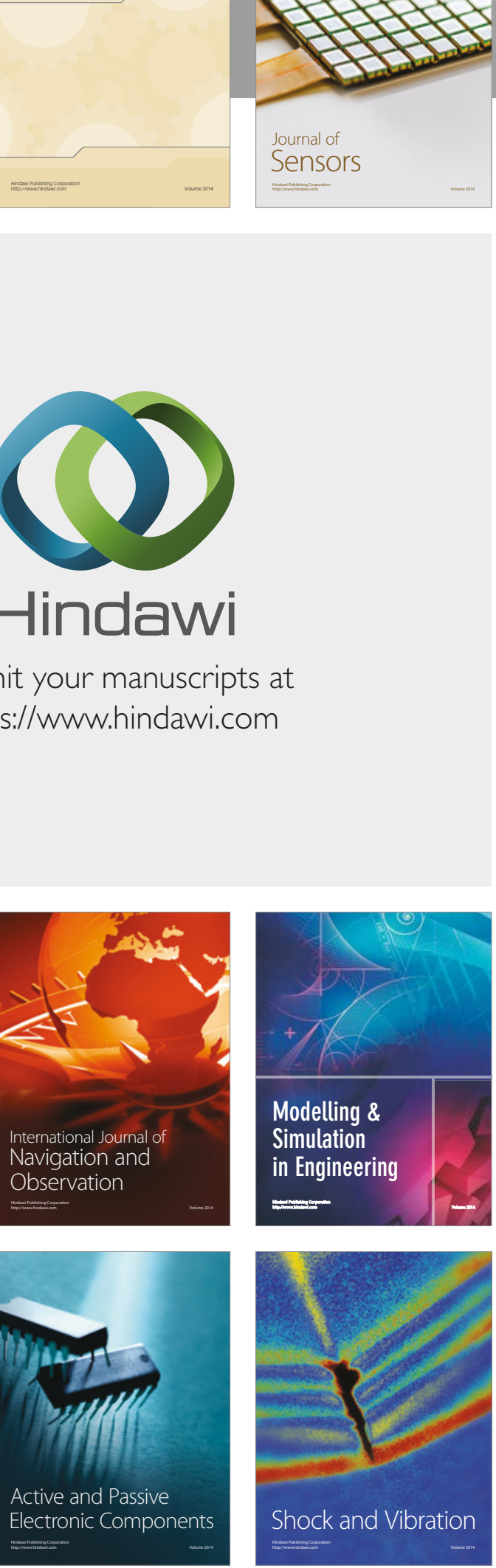
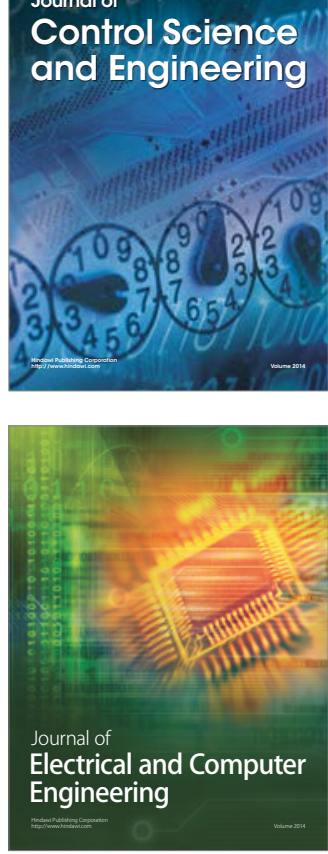

Distributed

Journal of

Control Science

and Engineering
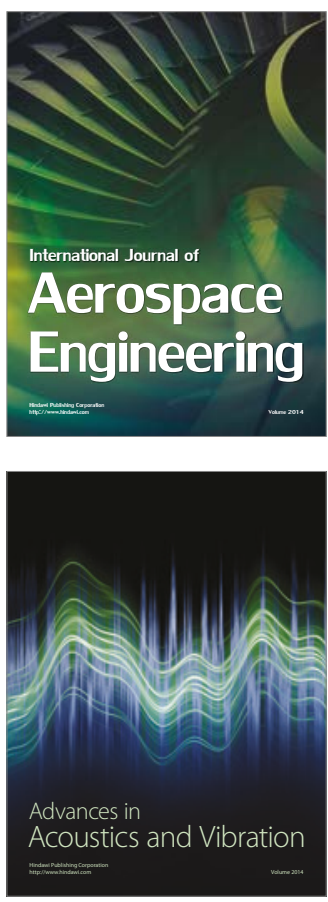

Sensor Networks 